\title{
Peer-to-peer cooperation for GPS positioning
}

\author{
Simone Morosi ${ }^{1,2, *, \dagger}$, Alessio Martinelli ${ }^{1,2}$ and Enrico Del Re $\mathrm{R}^{1,2}$ \\ ${ }^{1}$ Dipartimento di Ingegneria dell'Informazione, University of Florence, Florence, Italy \\ ${ }^{2}$ CNIT, University of Florence, Florence, Italy
}

\begin{abstract}
SUMMARY
This paper presents a peer-to-peer cooperative positioning technique together with its performance assessment. The cooperation between the two GPS receivers is realized by means of a wireless LAN connection and is significantly influenced by the accurateness of the synchronization between the two terminals. Both the outdoorto-indoor and outdoor-to-outdoor scenarios are considered. For each scenario, we assess the satellite signal acquisition and its computational load and the performance in terms of position accuracy and time-to-first-fix of the positioning procedure. Copyright (C) 2016 John Wiley \& Sons, Ltd.
\end{abstract}

Received 21 December 2014; Revised 18 April 2016; Accepted 26 May 2016

KEY WORDS: positioning; GNSS receiver; peer-to-peer; cooperative; software-defined implementation; synchronization

\section{INTRODUCTION}

Nowadays, the wider and wider spread of smart phones and mobile devices permits an impressive growth of location-based services with potential application in several areas [1-3], making positioning and navigation techniques one of the most popular research and development topics. Global navigation satellite system (GNSS) is the leader technology in outdoor positioning; it is characterized by global coverage that significantly increases the availability of the localization and enables an increasing adoption of related services. This trend will be even enforced by the upcoming multi-constellation system that involves several GNSS systems such as GPS, GLONASS, QZSS, Beidou and GALILEO. Unfortunately, even in outdoor scenarios, the pursue of an accurate position fix may become a complex issue in the presence of harsh environments such as dense urban canyons, light shadowing, or more generally when GNSS signals cannot be received with a sufficiently high signal-to-noise ratio (SNR). On the other hand, as for the indoor environments, many technologies may be adopted for localization purposes [4], but most of them requires a dedicated infrastructure, and their cost-effectiveness, reliability and standardization have still to be completely analyzed and assessed; moreover, an effective and seamless position service between outdoor and indoor positioning is still an open research issue at the moment. The cooperative positioning (CP) [5-9] system, which is represented in Figure 1, might be a solution to meet the positioning requirements such as accuracy and service availability through the cooperation between peers, especially in harsh environments where a stand-alone receiver cannot achieve the position fix.

In this paper, we present an overview about the state of the art of the $\mathrm{CP}$, focusing on the open points and the design and implementation challenges. Then, we describe the proposed peer-to-peer cooperative technique that has been proposed in [6]. Afterwards, we focus on the sensitivity-complexity tradeoff in the acquisition procedure, by comparing the effects of the Local Oscillator stability, the Doppler shift, and the correlation operation in both the stand-alone and cooperative cases. Then we take into account the synchronization issue, which may affect both the acquisition and the positioning routines.

* Correspondence to: Simone Morosi, University of Florence - CNIT Florence, Italy.

${ }^{\dagger}$ E-mail: simone.morosi@unifi.it 


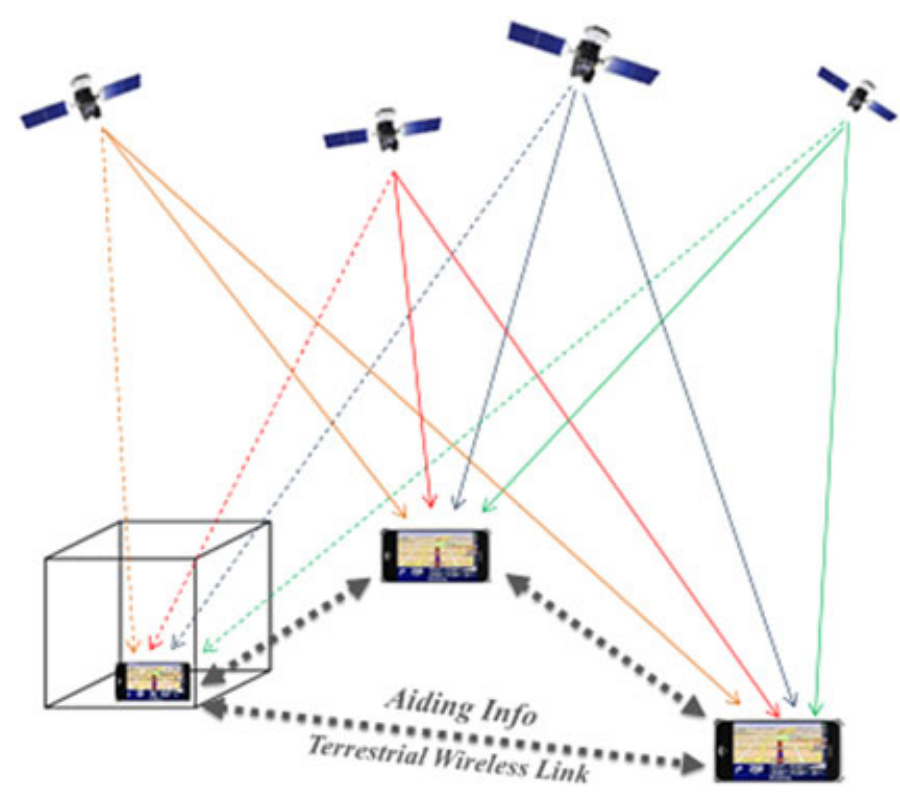

Figure 1. Cooperative positioning scenarios.

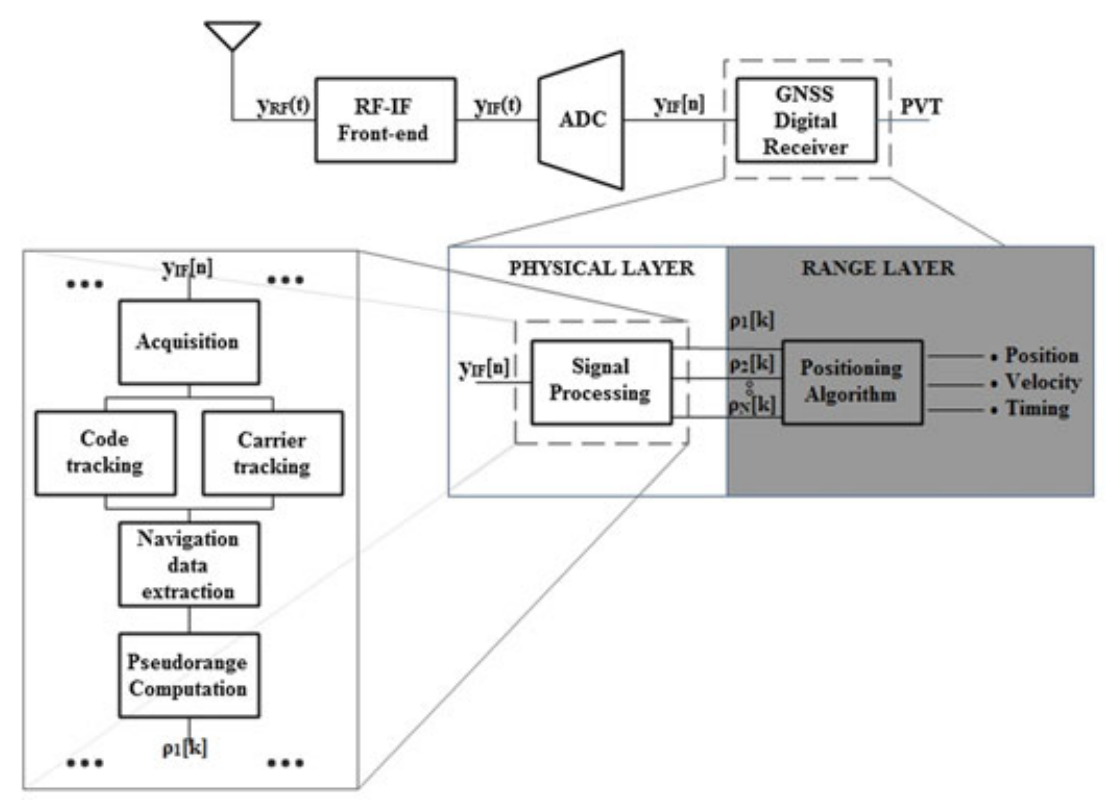

Figure 2. Stand-alone global navigation satellite system (GNSS) receiver representation.

Particularly, in the considered system the cooperation is carried out between two GPS software defined receivers that are both implemented in the MATLAB (Mathworks Inc., Natick, MA, USA) simulation environment, namely the aiding and aided receivers: the former is a stand-alone GPS software receiver [10], which performs the procedures, which are illustrated in Figure 2 and relies on the separation between physical and range layers, as for the general GNSS receiver; the latter is a GPS software defined receiver, which has been also implemented in MATLAB and performs the assisted acquisition and positioning algorithms with fine- or coarse-time assistance as defined by the synchronization level between the receivers. In the fine-time case, the common view [11] of at least one satellite is assumed for the aiding and aided terminals while a coarse-time assistance may be carried out by means of NTP protocol [12], that is to say by a software application, running on both the receivers. 
With respect to [13] and [14], the present work provides a new and wider set of results in addition to a general assessment of all the cooperative positioning features, which are evaluated in terms of satellite signal acquisition, computational load in the acquisition procedure, and performance concerning receiver position accuracy and time-to-first-fix (TTFF). The tests involve two scenarios: outdoor-toindoor $(\mathrm{O} 2 \mathrm{I})$ and outdoor-to-outdoor $(\mathrm{O} 2 \mathrm{O})$. The former is relative to a case in which the aiding and the aided receivers are located outdoor and indoor, respectively, whereas in the latter case both the receivers are located outdoor. The results highlight the cooperation benefits either in the O2I case where the localization accuracy improvements enable the aided receiver to get a position fix or in the $\mathrm{O} 2 \mathrm{O}$ scenario in which the aided receiver reduces the TTFF with respect to the stand-alone case.

\section{COOPERATIVE POSITIONING: STATE OF THE ART}

As anticipated in the Introduction, location based services are becoming very popular in the people's daily life, and most of the mobile applications rely on the device location information in order to access the context-aware services. At the same time, the positioning requirements in terms of service availability and accuracy are becoming more and more demanding. Therefore, in order to overcome the limitations of the stand-alone GNSS receiver and get a reliable position fix, innovative techniques have to be adopted: the CP paradigm might be a solution in some particular scenarios such as outdoor or dense urban areas as well as light indoor environments. In the CP context, two or more peers work together to improve their position information; in particular, the cooperation may exploit concepts such as proximity positioning [15], assisted/cooperative GNSS positioning [6, 13, 16-18], and opportunistic navigation [19]. Proximity positioning considers the user location as the position of the closest devices while the assisted GNSS positioning exploits the cellular network to provide data information to speed up the TTFF. On the other hand, cooperative GNSS positioning exploits peers that are close to each other, by resorting to the exchange of information and allowing the aided users, which are not in non-line of sight to determine their positions: with respect to the assisted GNSS, this technique better exploits the environment information and permits the aided device to improve the location accuracy. The concept of opportunistic navigation refers to the localization techniques, which exploit the signals of opportunity such as cellular, DVB, FM, AM, or Wi-Fi signals to obtain landmarks that are often registered through the received signal strength. In the cooperation context, it is possible to take advantage of the location map information when available and perform map matching algorithms to speed up the positioning procedure.

In the considered framework, an issue, which is still to be effectively addressed, is how to combine the previously cited concepts to improve the GNSS receiver performance in harsh environments where $\mathrm{CP}$ technology is not able to improve on the user positioning. This kind of integration might be limited by the traditional hardware-based devices, whereas a software-defined radio (SDR) approach can be an elegant solution to obtain a flexible architecture receiver and to allow a dynamic selection of parameters for the individual modules.

Moreover, the SDR implementation allows to effectively tackle the complexity of some specific tasks such as the satellite signal acquisition because this approach permits to easily replace the burdensome parallel correlation with a simple frequency domain multiplication [16]; particularly, the following relation holds

$$
(f(t) \star g(t))=\left(f^{*}(-t) \otimes g(t)\right)
$$

where the $\star$ and $\otimes$ symbols refer to the correlation and convolution operators, respectively. Hence, the correlation of the received signal with the PRN code can be substituted by a multiplication in the frequency domain, while the convolution result in the time domain can be obtained by an Inverse FFT (IFFT), as depicted in Figure 3. The convolution procedure in the frequency domain can be described by the following expression

$$
\begin{aligned}
z(t) & =\operatorname{IFFT}(X(j w) * Y(j w)) \\
& =\operatorname{IFFT}(F F T(x(t) \otimes y(t)))
\end{aligned}
$$




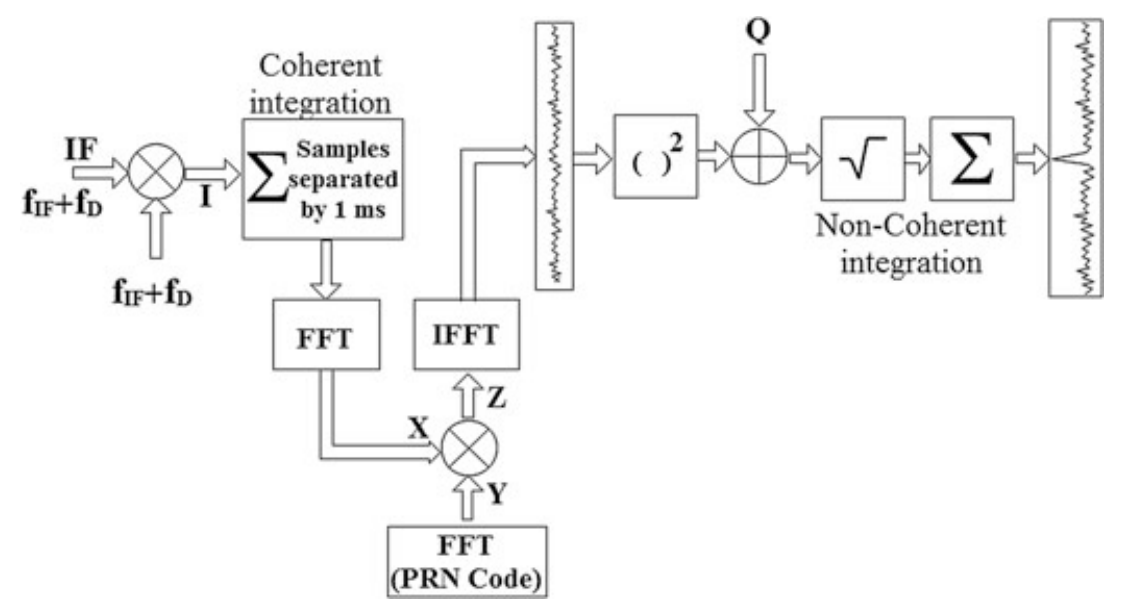

Figure 3. Acquisition scheme for software defined GNSS receiver using FFT implementation.

Another crucial CP issue is related to the fine-time synchronization between peers that are close to each other and connected by wireless networks. To this aim, even if the IEEE $802.11 \mathrm{v}$, which has been ratified as a formal amendment to the 802.11 standard in 2011, defines the basis for a fine-time synchronization in a Wi-Fi networi its use is still limited and not widespread.

\section{P2P GPS COOPERATIVE POSITIONING ALGORITHM}

The CP technique, which is proposed herein involves two GPS receivers whose cooperation is tested in the two scenarios, which are illustrated in Figure 1, namely the O2I and the O2O. The satellite signal acquisition and positioning algorithms that are running on the aided receiver and are implemented in the physical and range layers, respectively, will be affected by the accurateness of the time synchronization with the aiding terminal, leading to a fine or coarse time acquisition and positioning. The aiding information includes a combination of approximated user position, ephemerides, almanac, time and frequency assistance.

Concerning the signal acquisition procedure, the fine-time assistance is possible when the synchronization error between the aiding and the aided terminals is $<1 \mathrm{~ms}$ [16]. This condition permits the aided receiver either to speed the acquisition up by allowing the use of narrow search windows in the time domain or to increase its sensitivity by extending the coherent integration time during the process.

Regarding the positioning procedure, the time assistance is defined as fine when the time synchronization error is $<10 \mathrm{~ms}$ [16].

A GNSS receiver in LOS condition decodes the navigation message, which include the time-ofweek (TOW) information. The latter refers to the transmission time from the satellite and permits to form the complete pseudorange measurements. Conversely, if the received signal power is weak, as in the NLOS case (e.g. the receiver in light-indoor scenario), a GNSS terminal could not be able to correctly decode the TOW because of the high bit error rate and has to rely on external assistance to compute a reliable position fix. Furthermore, if the aided terminal is subject to a coarse-time assistance from the aiding one, it is not able to exploit the aiding data; particularly, because the satellites move at high velocity, the TOW value that is received by an aided terminal, which does not compensate the time error, leads to a wrong computation of the position of the satellites. ${ }^{\ddagger}$ Therefore, when only coarse-time assistance is available, the aided receiver has to estimate the synchronization error as an addition unknown in the navigation solution, that is, in addition to the usual four unknowns: user spatial coordinates and clock bias.

\footnotetext{
\$Because the maximum pseudorange rate of a GPS satellite is about $800 \mathrm{~m} / \mathrm{s}$, it can be noted that the satellite position computation that is based on a TOW estimate whose tolerance is less than $\pm 1 \mathrm{~s}$ would lead to a maximum geometric range error of 800 meters. User positions that are derived using such measurements can be mistaken by several kilometers [20].
} 
While the knowledge of the satellite velocity for each satellite can be obtained by the ephemeris information provided as external aiding information, the pseudorange rates for all satellites can be obtained from the relative velocities and included in the set of navigation equations together with the unknown position, the unknown receiver common bias, and the unknown coarse-time error. In order to understand this approach, it is worth considering how the residuals $\delta z$ of the of the a-priori pseudorange measurement is formed by the aided receiver [16]:

$$
\begin{gathered}
\delta z^{(k)}=z^{(k)}-\hat{z}^{(k)} \\
\hat{z}^{(k)}=\left|x^{(k)}\left(\hat{t}_{t x}\right)-x_{x y z 0}\right|-\delta t^{(k)}\left(\hat{t}_{t x}\right)+b_{0}
\end{gathered}
$$

where

- $z^{(k)}$ is the measured pseudorange relative to the $\mathrm{k}$-th satellite;

- $\hat{z}^{(k)}$ is the estimated pseudorange relative to the $\mathrm{k}$-th satellite;

- $\hat{t}_{t x}$ is the estimated time at which the satellite transmits the signal;

- $x^{(k)}\left(\hat{t}_{t x}\right)$ is the satellite position at time $\hat{t}_{t x}$;

- $x_{x y z 0}$ is the receiver a-priori position;

- $\delta t^{(k)}\left(\hat{t}_{t x}\right)$ is the satellite clock error at time $\hat{t}_{t x}$;

- $b_{0}$ is the a-priori estimate of the common bias.

If the error in the determination of the $\hat{t}_{t x}$ is not corrected by the aided receiver, it propagates to the values of $x^{(k)}, \delta t^{(k)}$, and $\hat{z}^{(k)}$ [19]. By computing the relative satellite-receiver velocities and the clock rate for all the satellites through the ephemeris information that is transmitted by an aiding terminal, we are able to compute the pseudorange rate, which can be exploited to determine the error in the $\hat{z}^{(k)}$ term caused by the time synchronization error in the provided a-priori time:

$$
\begin{aligned}
\hat{z}^{(k)}\left(\hat{t}_{t x}\right)-\hat{z}^{(k)}\left(t_{t x}\right) & =\hat{z}^{(k)}\left(\hat{t}_{t x}\right)-\hat{z}^{(k)}\left(\hat{t}_{t x}+\delta_{t c}\right) \\
& =-p^{(k)} * \delta_{t c}
\end{aligned}
$$

where

- $\delta_{t c}$ is the update to the coarse-time state;

- $t_{t x}$ is the 'true' time at which the satellite transmits the signal;

- $p^{(k)}=\left(e^{(k)} * v^{(k)}-\delta^{\prime}(k)\right)$ is the psudorange rate relative to the $\mathrm{k}$-th satellite;

- $v^{(k)}$ is the satellite velocity vector;

- $e^{k}$ is the unit vector from the receiver to the satellite $\mathrm{k}$;

By considering the relation between $z$ and $\delta_{t} c$ in (6), we can express (3) as follows:

$$
\delta z^{(k)}=z^{(k)}-\hat{z}^{(k)}=-e^{(k)} * \delta x_{x y z}+\delta b+p^{(k)} * \delta_{t c}+\epsilon^{(k)}
$$

where

- $\delta x_{x y z}=\left(\delta_{x}, \delta_{y}, \delta_{z}\right)$ is the update to the a-priori position;

- $\delta_{b}$ is the update to the a-priori receiver clock bias.

Thus, when dealing with coarse-time assistance, the geometry matrix is defined as

$$
H=\left(\begin{array}{ccc}
e^{(1)} & 1 & p^{(1)} \\
\vdots & \vdots & \vdots \\
e^{(k)} & 1 & p^{(k)}
\end{array}\right)
$$

If we assume at least five independent rows in $\mathrm{H}$, we can achieve $\delta \hat{x}$ (vector of updates to the a-priori aided receiver position) by using, for example, the standard least-squares solution: 


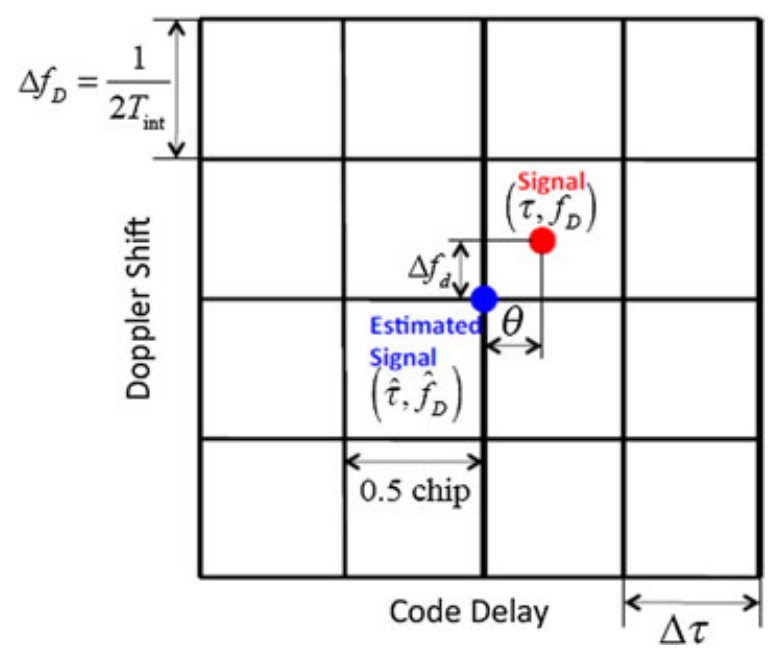

Figure 4. Acquisition code delay-frequency search-space.

$$
\delta \hat{x}=\left(H^{T} * H\right)^{-1} * \delta z=\left[\delta_{x}, \delta_{y}, \delta_{z}, \delta_{b}, \delta_{t c}\right]^{T}
$$

Hence, a close-form five-state solution to the position problem with no precise time is derived [16]: to this aim the following set of a-priori information is required:

- A valid set of ephemeris (in order to obtain satellite positions and their relative velocities);

- An approximation of the user position within a few kilometers;

- An approximation of the TOW within a few seconds.

In the P2P-CP case, the a-priori information, which is required for coarse-time positioning can be obtained by the aiding receiver. The sub-millisecond pseudorange can be computed by the aided receiver exploiting the aiding information that is provided at the physical layer. The integer-millisecond pseudorange has to be added to the submillisecond one to obtain an unambiguous pseudorange measurement. This operation has to be handled properly in order to avoid position errors resulting from a combination of sub-ms clock bias, measurement noise, and sub-ms pseudoranges. These combined values might exceed the one millisecond boundary, thus leading to rollovers. In [16, 21], an algorithm is described, which determines millisecond ambiguity.

The TOW estimation in the coarse-time navigation algorithms allows the following benefits:

- a faster TTFF;

- the achievement of a position fix under signal power conditions near or below data decoding threshold;

- the energy saving, which is due a remarkably shorter processing time to decode TOW and get a position fix, that is, some milliseconds wrt six or more seconds.

\section{ACQUISITION PROCEDURE: SENSITIVITY VS COMPLEXITY TRADE-OFF}

In this paragraph, the sensitivity and the complexity, which characterize the acquisition procedures are described and put in relation, with a particular attention to the trade-off between them. Both the stand-alone and the cooperative contexts are considered.

\subsection{Stand-alone positioning}

The signal acquisition that is performed by the GNSS receiver aims to determine a PRN code, a code delay $\tau$ and the Doppler offset $f_{D}$ of the signal, which has been transmitted by the satellites [22]. The correlation values that are determined by the IFFT block are commonly referred as cross ambiguity function $(\mathrm{CAF})$ between the incoming and the local generated signals [23, 24]. 


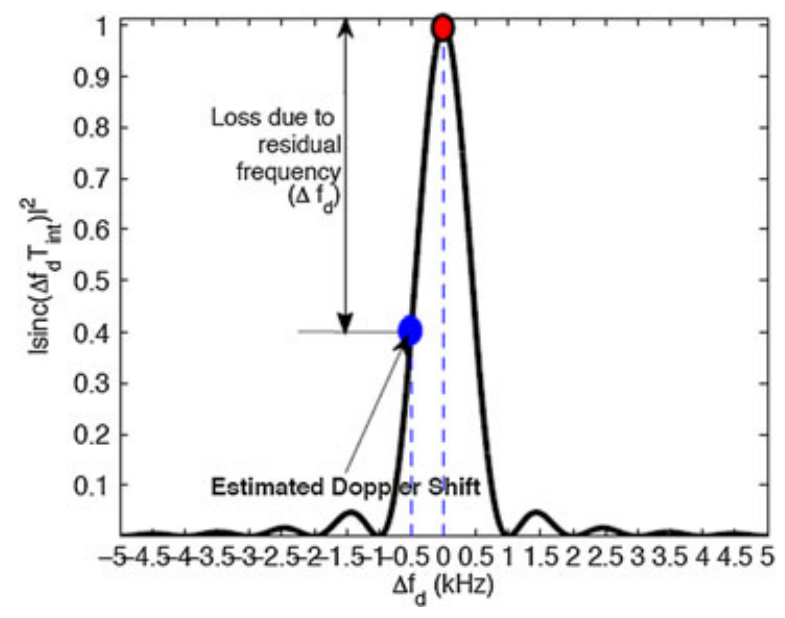

Figure 5. Sinc function describing the post-correlation SNR losses after the square function.

The CAF value is calculated for the time and the frequency bins in the discretized signal acquisition search space as depicted in Figure 4. Note that the $\Delta \tau$ and the $\Delta f_{D}$ bin widths determine the cell size in the time and frequency domains and therefore have to be chosen carefully in order to limit the cross-correlation loss. In particular, we focus on the frequency search space because the code delay may be sought by performing the correlation operation in the frequency domain as depicted in Figure 3 in order to reduce the complexity of the acquisition procedure in the time search space. Because the frequency bin width $\Delta f_{D}$ is inversely proportional to the acquisition procedure complexity, it has to be carefully defined; particularly, it has to be chosen together with the coherent integration time, which in turn is directly proportional to the sensitivity feature. Therefore, the function, which is described as

$$
\operatorname{sinc}\left(\Delta \bar{f}_{d_{m}} * T_{i n t}\right)=\frac{\sin \left(\Delta \bar{f}_{d_{m}} * T_{i n t}\right)}{\Delta \bar{f}_{d_{m}} * T_{i n t}}
$$

is responsible of post-correlation SNR losses, which affect the acquisition statistics where $\Delta \bar{f}_{d_{m}}=$ $f_{D}-\hat{f}_{D_{m}}$ is the difference between Doppler shifts during the interval $\mathrm{m}$ and $T_{i n t}$ is the coherent integration time; for clarity's sake, the function is depicted in Figure 5. Note that the $\left(\Delta \bar{f}_{d_{m}} * T_{i n t}\right)$ product brings out a complexity-sensitivity trade off: when the $T_{i n t}$ is increased, a post-correlation SNR raise (sensitivity augmentation) is observed, which leads to a frequency bin width reduction (complexity augmentation).

Note that the theoretical assessment of the acquisition performance is not always possible, because it requires also the knowledge of the pdf of the decision variables [23]; therefore, Monte-Carlo simulations are often employed to assess the acquisition schemes in different conditions; nonetheless, if the sensitivity of a single acquisition scheme in different conditions has to be evaluated, an easy-to-compute parameter such as the peak-to-floor ratio $\alpha_{\max }$ [23] can be used:

$$
\alpha_{\max }=\frac{\left|S_{\text {peak }}\right|^{2}}{\max \left|S_{\text {floor }}\right|^{2}}
$$

where $S_{\text {peak }}$ and $S_{\text {floor }}$ are the maximum and the floor of the CAF magnitude, respectively. This metric highlights the overall trend of the post-correlation SNR.

\subsection{Peer-to-peer cooperative positioning}

As for the acquisition, in the scenario, which is depicted in Figure 1, an aided receiver may increase its sensitivity or reduce the computational load depending on its position: if the receiver is located in a light-indoor situation, then an increase of the sensitivity will be pursued that will afford a correlation 


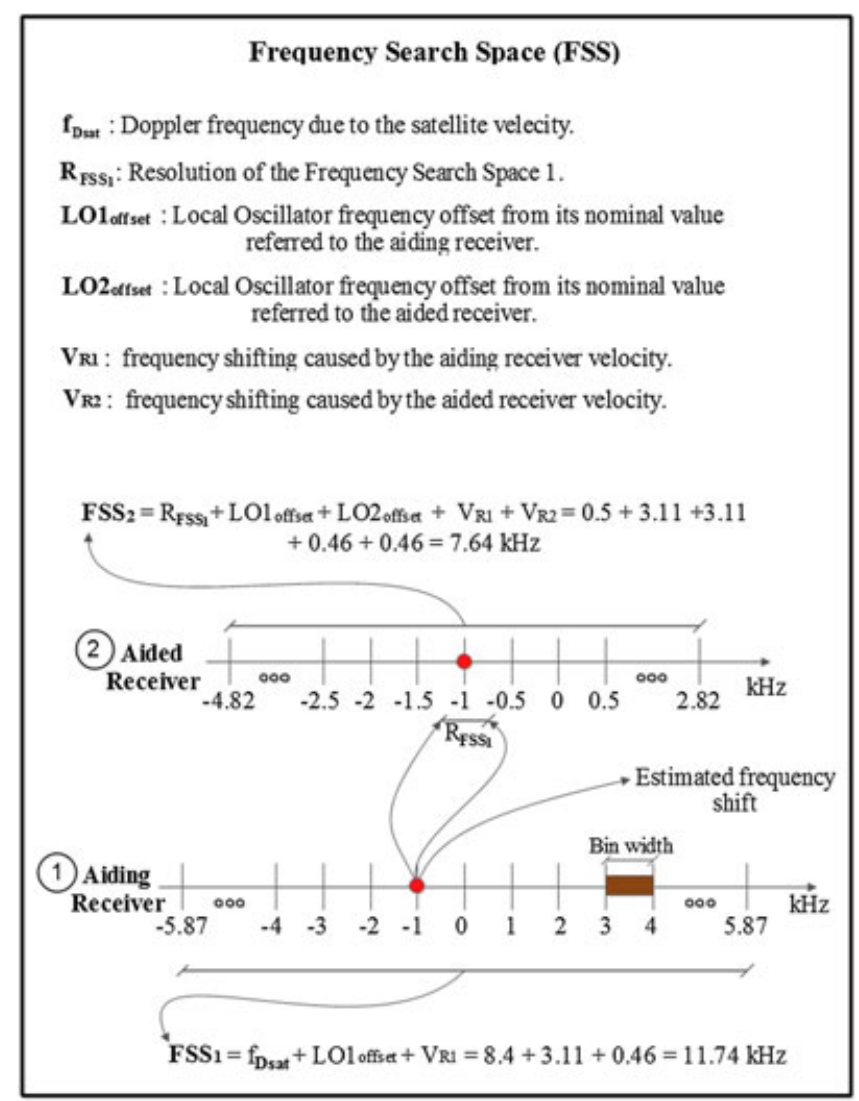

Figure 6. Frequency search space reduction for the aided receiver acquisition thanks to the Doppler information aid.

peak in the time-frequency search space with a sufficiently high value of $\alpha_{\max }(9)$; on the other hand, if the aided receiver is placed outdoor, a computational load reduction will be obtained by reducing the satellite signal acquisition time. A different sensitivity-complexity trade-off will be defined in the two situations by exploiting the information that is transmitted by an aiding receiver. In the case of sensitivity augmentation, the aided receiver generally increases the coherent integration time and performs non-coherent sums, as represented in the scheme in Figure 3. In this case, a reduction of the post-correlation SNR loss due to the $\operatorname{sinc}\left(\Delta \bar{f}_{d_{m}} T_{i n t}\right)$ term [23] will be achieved by lowering the frequency bin width $\Delta f_{D}$ in the frequency search space, as depicted in Figure 4. Unfortunately, the reduction may bring to a large number of frequency bins to be scanned, but, on the other hand, thanks to the Doppler information by the aiding receiver, the aided one can shrink the frequency search space and limit the number of bins. Conversely, in the case of computation load reduction, the aided receiver can leave unaltered the coherent integration time and the frequency bin width with respect to the aiding receiver and exploit the Doppler information aid to directly reduce the number of frequency bins in the acquisition search space. The example of Figure 6 shows the acquisition parameters [16] and illustrates how the frequency search space (FSS) can be reduced; thanks to the Doppler information aid. As illustrated in Figure 6, the aiding receiver $F S S_{1}$ is composed by the summation of the satellite Doppler $\left(f_{D_{s a t}}= \pm 4.2 \mathrm{kHz}\right)$, the term concerning the local oscillator frequency offset from its nominal value $\left(L O 1_{\text {offset }}= \pm 1.555 \mathrm{kHz}\right)$ and the frequency shift, which is caused by the receiver velocity $\left(V_{R 1}=\right.$ $\pm 0.23 \mathrm{kHz}$ ). The resulting $F F S_{1}$ is equal to $11.74 \mathrm{kHz}$. In this case, the acquisition correlation peak of the aiding receiver is assumed to be experienced at $-1 \mathrm{kHz}$. This value is transmitted to the aided receiver, which can reduce its $F F S_{2}$, which turns out to be the summation of the resolution of the $F F S_{1}= \pm 0.5 \mathrm{kHz}$, the terms concerning the local oscillator frequency offset from its nominal value referring to both the receivers $\left(L O 1_{\text {offset }}+L O 2_{\text {offset }}= \pm 1.555 \pm 1.555 \mathrm{kHz}\right)$ and the frequency shift that is originated by the velocities of both the receivers $\left(V_{R 1}+V_{R 2}= \pm 0.23 \pm 0.23 \mathrm{kHz}\right)$. As a result, the $F S S_{2}$ becomes equal to $7.64 \mathrm{kHz}\left(F S S_{2}=0.65 * F S S_{1}\right)$. 
In conclusion, we can assert that the $\mathrm{P} 2 \mathrm{P}-\mathrm{CP}$ is advantageous in the acquisition process for several reasons: the information that is provided by the aiding receiver allows the aided receiver to perform the acquisition process for a limited number of satellites, that is, the ones that are indicated by the aiding receiver and reduce the FSS with a time save in the acquisition procedure. Note that in order to limit the losses in the post-correlation SNR, a longer coherent integration time has to be balanced by a narrower frequency bin width, as described by [23] and depicted in Figure 5.

\section{TIME SYNCHRONIZATION}

Because CP is based on the exchange of time-sensitive information between peers, the time synchronization ends up being a fundamental issue to be dealt with. As described in the previous paragraph, the positioning algorithms, which are considered in this paper and developed for the assisted GPS receiver can resort to a fine- or a coarse-time synchronization. As far as this issue is concerned, the time synchronization requirements between the cooperative receivers are different depending on the considered operations, namely the Acquisition and the Positioning Algorithms [16], as represented in the scheme of Figure 2.

The acquisition procedure is considered to operate under fine-time synchronization if the time error between the clocks of the aiding and the aided receivers is less than $1 \mathrm{~ms}$; this choice allows to reduce the time search space in the aided receiver acquisition procedure. Because the considered acquisition algorithms rely on the use of the outputs of the FFT and the IFFT blocks, as shown in Figure 3, the fine-time synchronization is only considered for the identification of the bit transition in the navigation message: as a matter of facts, this operation permits to enhance the receiver sensitivity by increasing the coherent integration time. Moreover, the navigation message detection allows to compute the position of the satellites at the transmission time. Because the satellite-receiver range changes at a rate of up to $\pm 800 \mathrm{~m} / \mathrm{s}$, a time synchronization error of $10 \mathrm{~ms}$ may affect the satellite position with an error whose

Table I. Acquisition and positioning synchronization constraints.

\begin{tabular}{lcc}
\hline & Fine-time & Coarse-time \\
\hline Acquisition & $e r r_{\text {sync }}<1 \mathrm{~ms}$ & $e r r_{\text {sync }} \geq 1 \mathrm{~ms}$ \\
Positioning & $e r r_{\text {sync }}<10 \mathrm{~ms}$ & $e r r_{\text {sync }} \geq 10 \mathrm{~ms}$ \\
\hline
\end{tabular}

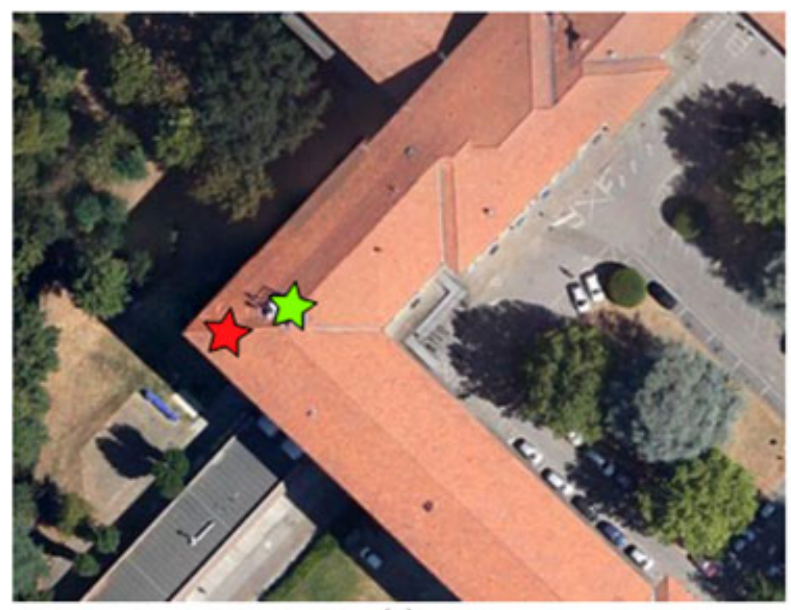

(a)

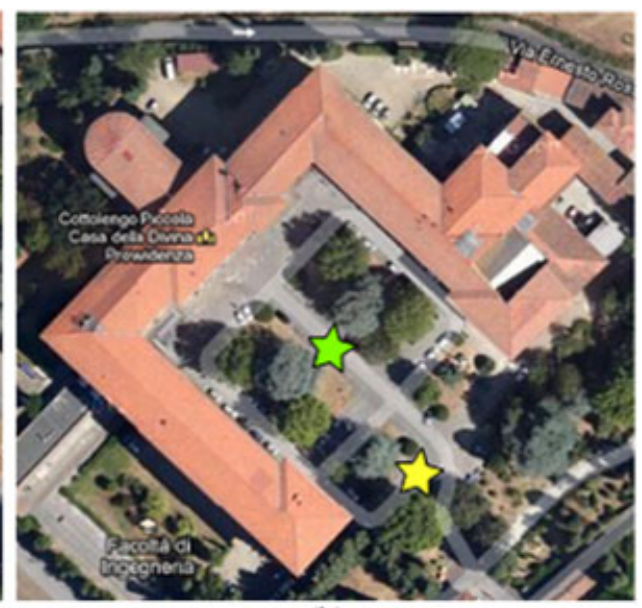

(b)

Figure 7. (a) Outdoor-to-indoor scenario. The green and red stars represent the aiding GPS receiver located outdoor and the indoor aided one, respectively. (b) Outdoor-to-outdoor scenario. Both the aiding (green star) and the aided (yellow star) receivers are located outdoor. 
value could be up to $8 \mathrm{~m}$. As a first fix is preferably within the range of acceptable accuracy, the $10 \mathrm{~ms}$ value is considered as a nominal cut-off point for precise time algorithm adoption in navigation [21, 25]. The Table I summarizes the time synchronization requirements for the aided receiver.

At the time being, the fine-time positioning synchronization is not possible through Wi-Fi protocol, until the new 802.11 chips implementing the amendment (v) will become available so permitting accurately synchronized clocks. On the other hand a software-based synchronization does not guarantee an accurate timing. In the present work, a fine-time synchronization is implemented assuming an initial common view [11] of at least one satellite. For what concerns coarse-time synchronization, network time protocol (NTP) may be a possible solution. NTP is one of the most popular protocols for internet time synchronization; it is used to synchronize the computer clocks within a network to a common reference; therefore, it allows to achieve synchronization between a trusted time-server and its clients [12]. On a local area network, NTP can achieve a precision of tens of milliseconds. The synchronization architecture uses a stratum concept [12], where each server on one level (stratum) is a time reference to lower levels. Primary servers are set at the root of the tree as stratum 1 and they are synchronized to external precise clock reference. Note that in Wireless LAN, multipath effects may contribute to the physical level jitter; therefore, when dealing with WLAN, the synchronization protocols may show worse performance than in wired LAN.

\section{EXPERIMENTAL SETUP}

As anticipated in the previous paragraphs, the peer-to-peer cooperative algorithm with coarse/finetime acquisition and positioning is implemented on GPS software-defined receivers. The cooperation is carried out between two close GPS software receivers, namely the aiding and the aided receivers. The aiding receiver is a stand-alone GPS software receiver, which is implemented in MATLAB [10]; it is able to perform acquisition (through a fast parallel acquisition technique, in which the correlation function is evaluated by means of FFT operations), code and carrier tracking, navigation bit extraction, navigation data decoding, pseudorange estimations, and position computations. Also the aided GPS receiver is implemented in MATLAB and performs assisted acquisition and positioning algorithms with fine/coarse-time assistance. In this experiment, both the software receivers run over common laptops and process data, which are sampled at $16.368 \mathrm{MHz}$. The sampled data are achieved from a front-end module, that is, SiGe GN3S v3 [26], which is connected via USB to the laptops. The front-end processes the satellite signals (L1 GPS signal at $1575.42 \mathrm{MHz}$ ) that is received by a GPS patch antenna; a sampled output is provided after filter, amplifier, mixer and ADC operations. The receivers are connected through an ad-hoc Wireless LAN that allows to exchange assistance and synchronization data. In the coarse-time context, both the receivers run a background application implementing an NTP protocol, which is used for coarse synchronization. The aiding receiver is a time server while the aided one is considered to be the client. The scenarios, which are considered in this paper are illustrated in Figure 7 . Particularly, Figure 7(a) represents the O2I case; the aiding receiver is located outdoor (above the roof top), about $8 \mathrm{~m}$ far from the aided receiver, which is located indoor (below the roof top). On the other hand, the Figure 7(b) illustrates the $\mathrm{O} 2 \mathrm{O}$ scenario, where both the aiding and the aided receivers are located outdoor in LOS condition, at a mutual distance of about $30 \mathrm{~m}$. Four tests will be performed for each $\mathrm{O} 2 \mathrm{I} / \mathrm{O} 2 \mathrm{O}$ scenario; moreover, two synchronization time levels will be considered for each test: the former refers to an off-line ideal synchronization between the aiding and the aided receivers in the context of fine-time assistance, while the latter considers the coarse-time assistance, that is, the receivers are synchronized by means of the NTP protocol.

\section{EXPERIMENTAL RESULT}

The results of the performed acquisition and positioning tests are described in Tables II, III, IV, and V. Each table refers to a particular satellite configuration and to specific $\mathrm{O} 2 \mathrm{I} / \mathrm{O} 2 \mathrm{O}$ scenarios and illustrates the pseudo-random noise sequences and the SNR levels that are relative to the satellite geometries which are acquired by the aided receiver. The descriptions of the positioning errors in the east and north directions are also shown; it is worth highlighting that 100 trials have been performed for each 
Table II. Aided receiver acquisition and positioning results - test no. 1 .

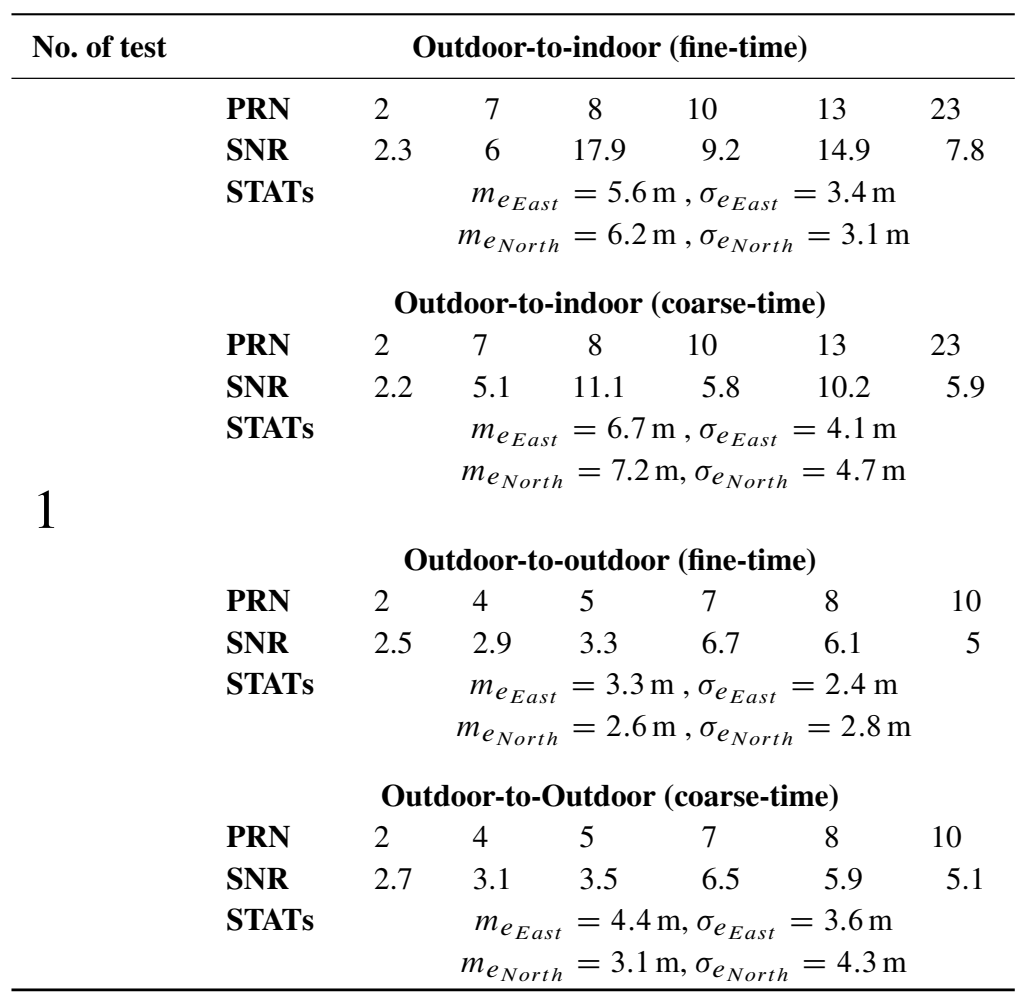

Table III. Aided receiver acquisition and positioning results - test no. 2 .

\begin{tabular}{|c|c|c|c|c|c|c|}
\hline \multirow[t]{2}{*}{ No. of test } & \multicolumn{6}{|c|}{ Outdoor-to-indoor (fine-time) } \\
\hline & PRN & 2 & 12 & 24 & 25 & 29 \\
\hline \multirow{14}{*}{2} & SNR & 5.5 & 15.5 & 2.4 & 16.2 & 6.7 \\
\hline & STATs & \multicolumn{5}{|c|}{$\begin{aligned} m_{e_{\text {East }}} & =6.1 \mathrm{~m}, \sigma_{e_{\text {East }}}=3.2 \mathrm{~m} \\
m_{e_{\text {North }}} & =7.1 \mathrm{~m}, \sigma_{e_{\text {North }}}=3.8 \mathrm{~m}\end{aligned}$} \\
\hline & \multicolumn{6}{|c|}{ Outdoor-to-indoor (coarse-time) } \\
\hline & PRN & 2 & 12 & 24 & 25 & 29 \\
\hline & SNR & 4.1 & 11.2 & 2.1 & 12.9 & 5.6 \\
\hline & STATs & \multicolumn{5}{|c|}{$\begin{aligned} m_{e_{\text {East }}} & =7.7 \mathrm{~m}, \sigma_{e_{\text {East }}}=4.9 \mathrm{~m} \\
m_{e_{\text {North }}} & =8.1 \mathrm{~m}, \sigma_{e_{\text {North }}}=5.2 \mathrm{~m}\end{aligned}$} \\
\hline & \multicolumn{6}{|c|}{ Outdoor-to-outdoor (fine-time) } \\
\hline & PRN & 12 & 13 & 15 & 17 & 24 \\
\hline & SNR & 4.8 & 3.9 & 4.2 & 3.1 & 7.3 \\
\hline & STATs & \multicolumn{5}{|c|}{$\begin{aligned} m_{e_{\text {East }}} & =3.9 \mathrm{~m}, \sigma_{e_{\text {East }}}=2.8 \mathrm{~m} \\
m_{e_{\text {North }}} & =3.3 \mathrm{~m}, \sigma_{e_{\text {North }}}=2.9 \mathrm{~m}\end{aligned}$} \\
\hline & \multicolumn{6}{|c|}{ Outdoor-to-outdoor (coarse-time) } \\
\hline & PRN & 12 & 13 & 15 & 17 & 24 \\
\hline & SNR & 4.3 & 4.2 & 5.3 & 3.9 & 7.6 \\
\hline & STATs & \multicolumn{5}{|c|}{$\begin{aligned} m_{e_{\text {East }}} & =4.7 \mathrm{~m}, \sigma_{e_{\text {East }}}=4.1 \mathrm{~m} \\
m_{e_{\text {North }}} & =4.5 \mathrm{~m}, \sigma_{e_{\text {North }}}=4.4 \mathrm{~m}\end{aligned}$} \\
\hline
\end{tabular}


Table IV. Aided receiver acquisition and positioning results - test no. 3 .

\begin{tabular}{|c|c|c|c|c|c|c|c|}
\hline \multirow[t]{4}{*}{ No. of test } & \multicolumn{7}{|c|}{ Outdoor-to-indoor (fine-time) } \\
\hline & PRN & 14 & 18 & 19 & 21 & 22 & 27 \\
\hline & SNR & 2.3 & 9.5 & 7.5 & 2.4 & 12.5 & 15.9 \\
\hline & STATs & & $\begin{array}{r}m_{e_{E a}} \\
m_{e_{N o r t}}\end{array}$ & $\begin{array}{l}=4.9 \\
=5.5\end{array}$ & $\begin{array}{l}\sigma_{e_{\text {East }}} \\
\sigma_{e_{\text {Nort }}}\end{array}$ & $\begin{array}{l}2.8 \mathrm{~m} \\
=3.5 \mathrm{~m}\end{array}$ & \\
\hline \multirow{12}{*}{3} & \multicolumn{7}{|c|}{ Outdoor-to-indoor (coarse-time) } \\
\hline & PRN & 14 & 18 & 19 & 21 & 22 & 27 \\
\hline & SNR & 2.1 & 7.5 & 4.2 & 2 & 8.3 & 11.1 \\
\hline & STATs & \multicolumn{6}{|c|}{$\begin{aligned} m_{e_{\text {East }}} & =6.2 \mathrm{~m}, \sigma_{e_{\text {East }}}=3.6 \mathrm{~m} \\
m_{e_{\text {North }}} & =7.2 \mathrm{~m}, \sigma_{e_{\text {North }}}=3.9 \mathrm{~m}\end{aligned}$} \\
\hline & \multicolumn{7}{|c|}{ Outdoor-to-outdoor (fine-time) } \\
\hline & PRN & 4 & 11 & 19 & 22 & 27 & 32 \\
\hline & SNR & 3.5 & 2.8 & 7.4 & 4.1 & 4.9 & 5.3 \\
\hline & STATs & \multicolumn{6}{|c|}{$\begin{aligned} m_{e_{\text {East }}} & =1.9 \mathrm{~m}, \sigma_{e_{\text {East }}}=3.1 \mathrm{~m} \\
m_{e_{\text {North }}} & =2.6 \mathrm{~m}, \sigma_{e_{\text {North }}}=2.5 \mathrm{~m}\end{aligned}$} \\
\hline & \multicolumn{7}{|c|}{ Outdoor-to-outdoor (coarse-time) } \\
\hline & PRN & 4 & 11 & 19 & 22 & 27 & 32 \\
\hline & SNR & 3.9 & 3.5 & 7.9 & 2.9 & 5.6 & 4.5 \\
\hline & STATs & \multicolumn{6}{|c|}{$\begin{aligned} m_{e_{\text {East }}} & =3.5 \mathrm{~m}, \sigma_{e_{\text {East }}}=2.9 \mathrm{~m} \\
m_{e_{\text {North }}} & =3.8 \mathrm{~m}, \sigma_{e_{\text {North }}}=3.1 \mathrm{~m}\end{aligned}$} \\
\hline
\end{tabular}

Table V. Aided receiver acquisition and positioning results - test no. 4 .

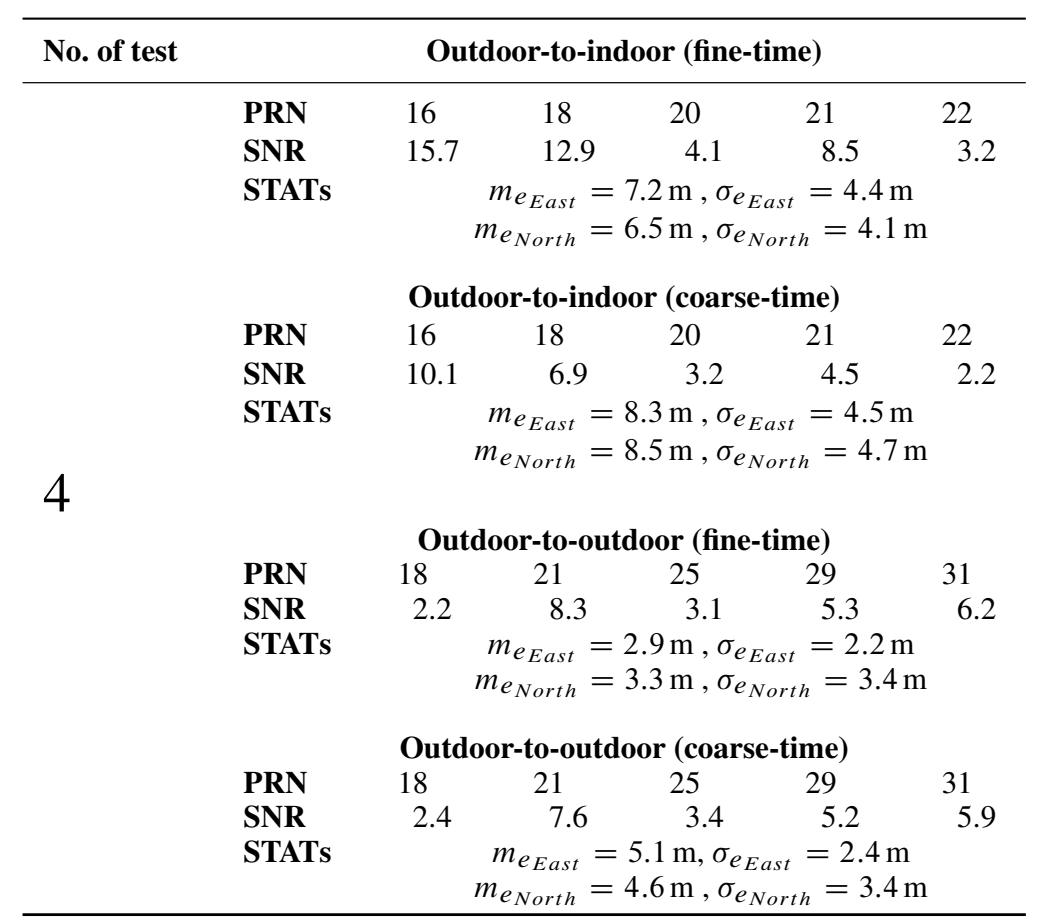

configuration and that the average of the obtained results are presented. For sake of space, the following subsections only refer to the first tests of Table II and are used to assess the satellite signal acquisition, the computational load in the acquisition procedure and the performance concerning the receiver position accuracy and time-to-first-fix. 


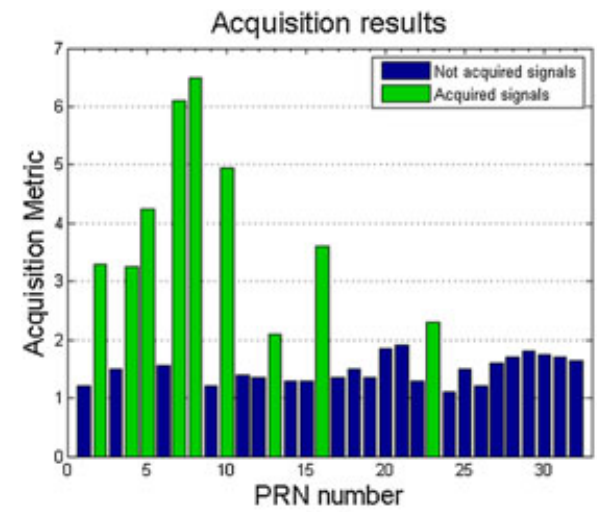

(a)

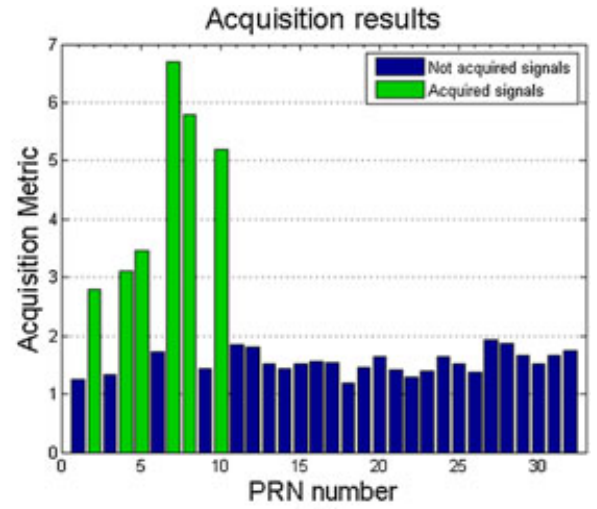

(b)

Figure 8. (a) Aiding receiver acquisition results, $1 \mathrm{~ms}$ coherent integration, one non-coherent integration, O2I scenario. (b) Aiding receiver acquisition results, $1 \mathrm{~ms}$ coherent integration, one non-coherent integration, $\mathrm{O} 2 \mathrm{O}$ scenario.

\subsection{Satellite signal acquisition assessment}

7.1.1. Outdoor-to-indoor (O2I) aiding scenario. The aiding receiver performs acquisition and tracking in the stand-alone mode; the acquisition results are shown in Figure 8(a).

The receiver acquires the $2,4,5,7,8,10,13,16$, and 23 satellites by means of an $1 \mathrm{~ms}$ coherent integration and of one non-coherent integration. The green bars in Figure 8(a) refer to the acquired satellites. Each single peak represents the ratio between the highest and second highest peak in the correlation performed over the code delay-frequency search space for each satellite.

Assuming fine-time assistance condition, the aided receiver performs an assisted acquisition; thanks to the following information, which is broadcasted by the aiding receiver:

- Satellite IDs for the visible satellites;

- Doppler frequency relative to each satellite (to reduce the frequency search space in the acquisition process);

- Time stamp of the first sub-frame of the navigation message (to identify the bit transition time).

The aided receiver will try to acquire only the satellites, which are indicated by the aiding receiver. As shown in Figure 9(a), it is not able to acquire satellites with a $1 \mathrm{~ms}$ coherent integration and one noncoherent integration because the received signal is weak because of the indoor location; particularly, the post-correlation procedure that is illustrated in Figure 3 produces a value of $n-\alpha_{\max }$ (9) that is lower than the threshold $\alpha_{T s h}=2$, which has been chosen as a design parameter and used for all the considered satellites. Therefore, the receiver sensitivity has to be improved.

In the fine-time acquisition case, the aided receiver gets to know precisely when the bit transitions of the navigation message occur. Thus, it can wipe off the signal from the navigation message end extend the coherent integration period to increase the receiver sensitivity. Hence, by considering $100 \mathrm{~ms}$ as the overall integration time ${ }^{\S}$ the coherent integration time is set equal to $20 \mathrm{~ms}$ while 5 non-coherent integrations are performed; as a result, the $\alpha_{\max }>2$ constraint is respected for all the six satellites that are identified in Figure 10(a).

In this study, the $\operatorname{sinc}\left(\Delta \bar{f}_{d_{m}} T_{i n t}\right)$ term has been defined by assuming that $\Delta f_{D}=\frac{1}{2 T_{i n t}}$; hence, the loss factor is less than or equal to $|\operatorname{sinc}(0.5)|^{2}=0.4$. The acquisition results with the new integration parameters are shown in Figure 10(a).

When a fine-time synchronization cannot be realized, the acquisition procedure has to resort to a coarse-time approach. In this case, the aiding receiver performs the same tasks as in the fine-time

\footnotetext{
$\S_{\mathrm{A}} 100 \mathrm{~ms}$ integration time is chosen because during this long interval the satellite-receiver dynamic changes are not considerably affecting the signal acquisition.
} 


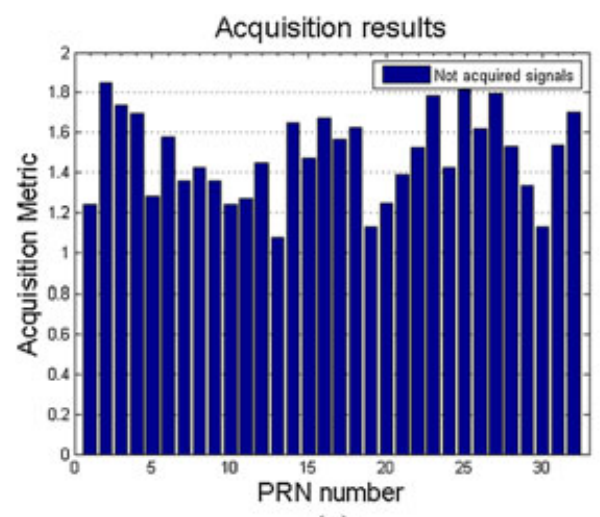

(a)

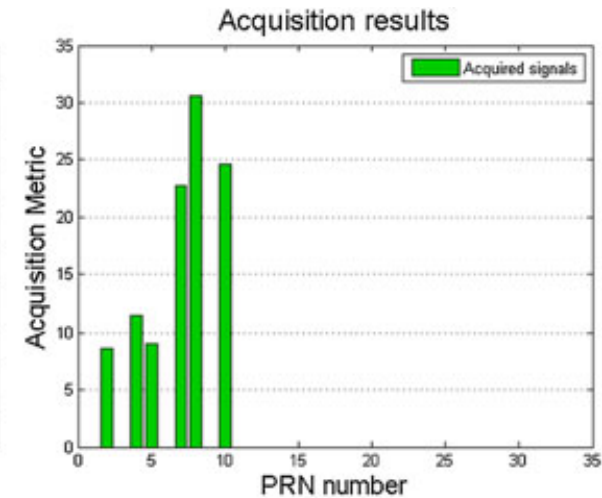

(b)

Figure 9. (a) Aided receiver acquisition results, $1 \mathrm{~ms}$ coherent integration, one non-coherent integration, O2I scenario. (b) Aided receiver acquisition results, $1 \mathrm{~ms}$ coherent integration, one non-coherent integration, $\mathrm{O} 2 \mathrm{O}$ scenario.

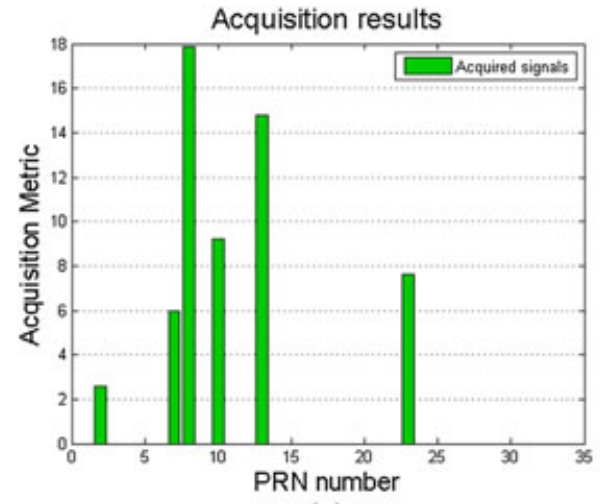

(a)

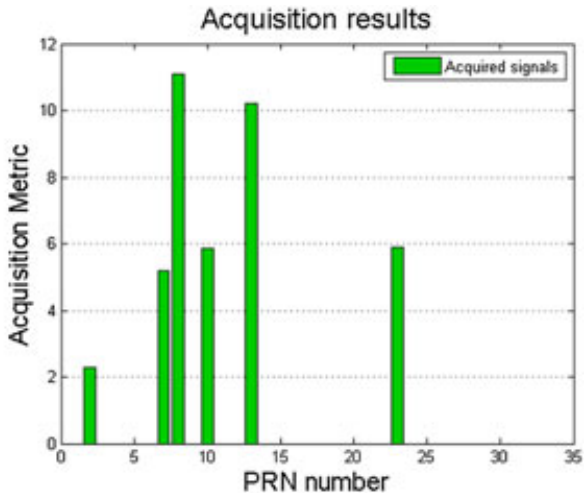

(b)

Figure 10. (a) Aided receiver acquisition results, $20 \mathrm{~ms}$ coherent integration, five non-coherent integrations, finetime assistance, O2I scenario. (b) Aided receiver acquisition results, $11 \mathrm{~ms}$ of coherent integration, nine noncoherent integrations, coarse-time assistance, O2I scenario.

assistance case; the main difference concerns the aiding information; in this case, the time stamp of the first sub-frame of the navigation message is not broadcasted. Therefore, the coherent integration time of the aided receiver has to be extended to a shorter interval than in the fine-time assistance because of the uncertainty about the bit transitions in the navigation message. In the signal acquisition routine, the coherent integration time has been set equal to $11 \mathrm{~ms}$ while nine non-coherent sums are considered to keep a total integration time of about $100 \mathrm{~ms}$. Once again, the coherent integration procedure leads to a post-correlation loss factor equal to 0.4 because the $\left(\Delta \bar{f}_{d_{m}} T_{i n t}\right)$ product is unchanged with respect to the fine-time case. The acquisition results are shown in Figure 10(b).

7.1.2. Outdoor-to-outdoor $(\mathrm{O} 2 \mathrm{O})$ aiding scenario. The aided receiver acquisition procedure does not need to take advantage of fine-time assistance to increase its sensitivity; this is not surprising since, thanks to the LOS condition of the satellites, the aided receiver does not need an extension of the coherent integration time. As described in the example depicted in Figure 6, the aided receiver can directly exploit the Doppler information to reduce the number of bins and, consequently, the complexity of the receiver acquisition. It is important to note that the Doppler information that is provided by the aiding receiver is related to the resolution of the frequency search space that is determined by the acquisition and illustrated in Figure 6. In all the considered tests, the resolution of the aiding receiver frequency search space is equal to $500 \mathrm{~Hz}$, which becomes the initial space in the aided receiver frequency search space. 
In both fine- and coarse-time assistance cases, the aiding receiver performs acquisition and tracking in stand-alone mode with the results that are depicted in Figure 8(b); by means of $1 \mathrm{~ms}$ coherent and 1 non-coherent integrations, the receiver acquires the $2,4,5,7,8$, and 10 satellites. The aided receiver performs the assisted acquisition; thanks to the received information, which is the same of the O2I scenario. The integration parameters are equal to $1 \mathrm{~ms}$ for both the coherent and the non-coherent parts. The acquisition results are shown in Figure 9(b).

\subsection{Computational load assessment in the signal acquisition procedure}

7.2.1. Outdoor-to-indoor (O2I) aiding scenario. In order to compare the computational load of the acquisition of both the aided and the aiding receivers, we consider the number of bins in the frequency search space. The overall frequency search space in the aided acquisition is defined by the following parameters:

- assistance reference-frequency uncertainty, which is given by the sum of the frequency search space resolution $\left(R_{F S S \text {-aiding }}\right)$ and the local oscillator frequency offset $\left(L O_{\text {offset-aiding }}\right)$, both referred to the aiding receiver case;

- local oscillator frequency offset of the aided receiver ( $\left.L O_{\text {offset-aided }}\right)$;

- time assistance;

- a-priori position (aiding receiver position);

- Doppler frequency due to the satellite-aiding/aided receiver relative velocity.

We can ignore the last three uncertainties by assuming that the receivers are not moving and their distance is less than $1 \mathrm{~km}$ [16]. Considering the tolerance of the front-end TCXO (Temperature Compensated Crystal Oscillator) equal to $\pm 1 \mathrm{ppm}$ either for the aided or aiding receiver, the aided receiver frequency search space ( $\left.F S S_{\text {aided }}\right)$ is given by

$$
\begin{aligned}
F S S_{\text {aided }}= & R_{F S S \text {-aiding }}+L O_{\text {offset-aiding }} \\
& +L O_{\text {offset-aided }}=0.5+3.11+3.11 \\
= & 6.72 * 10^{3} \mathrm{kHz}
\end{aligned}
$$

Hence, in the fine-time synchronization case, the frequency bin width in the acquisition frequency search space of the aided receiver can be determined as

$$
F W=\frac{\frac{1000}{C I T}}{2}=\frac{\frac{1000}{20}}{2}=25 \mathrm{~Hz}
$$

where CIT is the coherent integration time and FW is the frequency width. Therefore, the number of frequency bins for the aided receiver acquisition is

$$
\begin{aligned}
& N_{\mathrm{BIN}}(\text { Aided Receiver })_{\text {Fine-time }}=\frac{F S S_{\text {aided }}}{F W}= \\
& =\frac{6.72 * 10^{3}}{25}=268 \text { bins. }
\end{aligned}
$$

On the other hand, the number of frequency bins for the aiding receiver acquisition is equal to

$$
\begin{aligned}
& N_{\mathrm{BIN}}(\text { Aiding Receiver })_{\text {Fine-time }}=\frac{F S S_{\text {aiding }}}{F W}= \\
& =\frac{8.4 * 10^{3}+3.1 * 10^{3}}{500}=23 \text { bins, }
\end{aligned}
$$

where $8.4 * 10^{3} \mathrm{~Hz}$ is the frequency range due to the Doppler effect of satellite motion and $500 \mathrm{~Hz}$ is the frequency bin width resulting from 1-ms coherent integration. For what concerns the computation load, by taking into account the number of bins to be scanned during the acquisition procedure, we can conclude that the aided receiver acquisition is about 11 times more complex than the aiding one. 
In the case of coarse-time synchronization, the number of frequency bins in the search space of the aiding receiver acquisition is equal to 23 , as in the fine-time assistance test. Conversely, the number of frequency bins for the aided receiver is

$$
\begin{aligned}
& N_{\mathrm{BIN}}(\text { Aided Receiver })_{\text {Coarse-time }}=\frac{F S S_{\text {aided }}}{F W}= \\
& =\frac{6.72 * 10^{3}}{45}=150 \text { bins, }
\end{aligned}
$$

where $45 \mathrm{~Hz}$ is the frequency bin width, which is defined by the choice of the coherent integration equal to $11 \mathrm{~ms}$. By considering the number of bins to be scanned during the acquisition process, we can conclude that the aided receiver acquisition lasts about six times more than in the aiding one.

7.2.2. Outdoor-to-outdoor $(\mathrm{O} 2 \mathrm{O})$ aiding scenario. If a 1 -ms coherent-time integration and 1-ms noncoherent integration are considered, the number of frequency bins for the aided receiver acquisition, either in the fine or in the coarse-time assistance case, is computed as follows:

$$
\begin{aligned}
& N_{\mathrm{BIN}}(\text { Aided Receiver })_{\text {Fine/Coarse-time }}=\frac{F S S_{\text {aided }}}{F W}= \\
& =\frac{6.72 * 10^{3}}{500}=13 \text { bins, }
\end{aligned}
$$

where $500 \mathrm{~Hz}$ is the frequency bin width resulting from the 1-ms coherent integration time. The number of bins in the aiding receiver acquisition can be considered the same as in (12). In conclusion, in the $\mathrm{O} 2 \mathrm{O}$ aiding scenario, the aided receiver acquisition time is about two times smaller than the aiding receiver acquisition one.

\subsection{Performance assessment: position accuracy and time-to-first fix}

7.3.1. Outdoor-to-indoor (O2I) aiding scenario. While the aiding receiver performs the usual standalone positioning procedure, the aided one takes benefit from the following information that is broadcasted by the aiding terminal:

- position of the aiding receiver;

- navigation message (Ephemeris and almanac in order to compute the satellite position at the transmission time, ionospheric model and satellite clock corrections).

Once the aided receiver has accomplished the acquisition, the sub-ms pseudoranges are available. After the integer millisecond ambiguity is eliminated, the complete pseudoranges for the aided receiver are determined together with the satellite position at the transmission time thanks to the ephemeris knowledge.

If fine-time assistance is guaranteed, the position of the aided receiver is determined by means of Extended Kalman Filter (EKF) method (using the position of the aiding terminal as a-priori position [27]). The positioning procedure results are shown in Figure 11(a), while the statistical description (STATs) that are represented in Table II reports the mean error and the standard deviation, which are relative to East and North axes.

On the other hand, when the coarse-time synchronization takes place, the aiding receiver performs the positioning procedure in a stand-alone mode and broadcasts the same aiding quantities to the aided receiver as in the fine-time assistance case. After the aiding information has been received, the aided receiver computes the complete pseudoranges; then, because coarse-time assistance is supposed, the time error has to be compensated to avoid satellite position errors. The compensation is performed by computing the pseudorange rates (for each satellite-receiver link) and including them in the geometry matrix [16]; afterwards, the five-state updates are computed using the EKF method. The position results are shown in Figure 11(b), while Table II reports the performance, which is relative to East and North axes in terms of mean error and standard deviation. The position error is larger than in the fine-time assistance case mostly because of two reasons: 


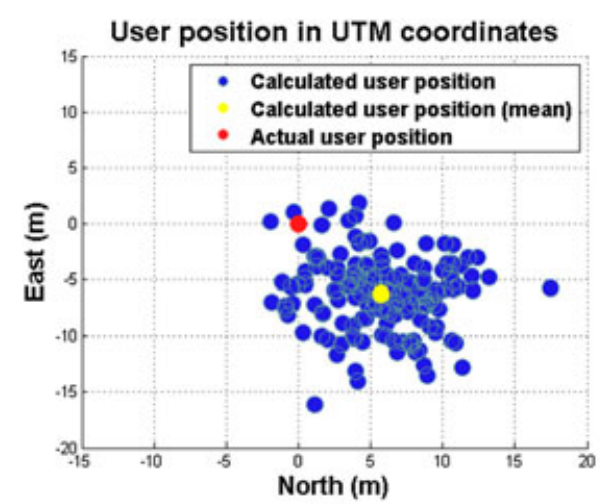

(a)

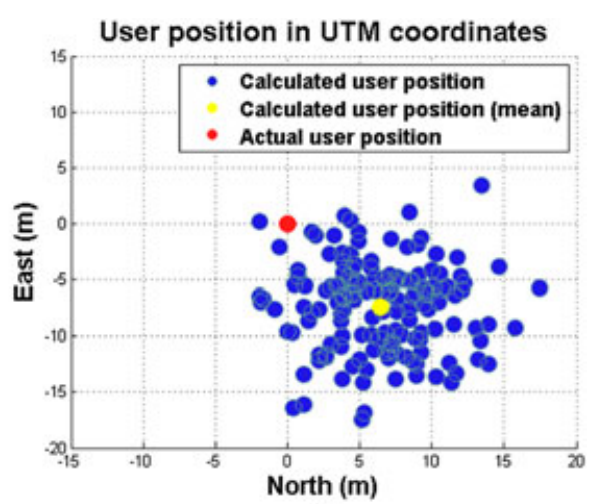

(b)

Figure 11. (a) Aided receiver position with fine-time assistance in O2I scenario. (b) Aided receiver position with coarse-time assistance in $\mathrm{O} 2 \mathrm{I}$ scenario.

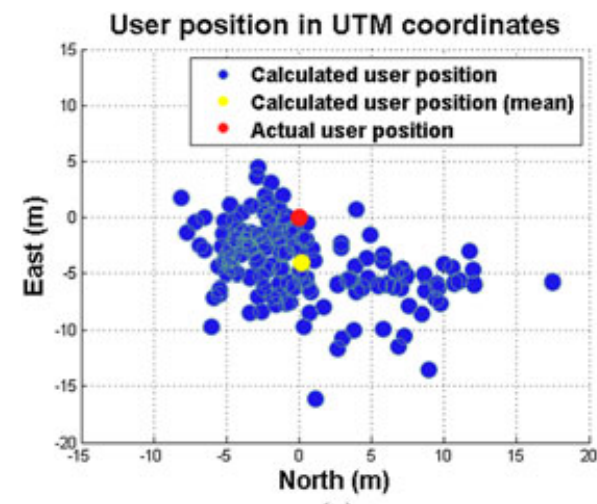

(a)

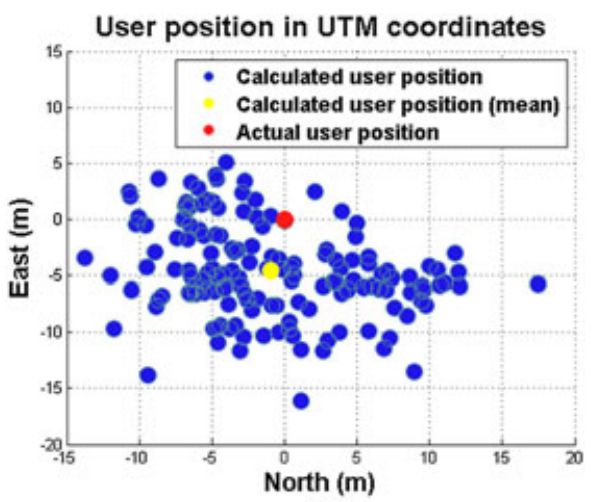

(b)

Figure 12. (a) Aided receiver position with fine-time assistance in $\mathrm{O} 2 \mathrm{O}$ scenario. (b) Aided receiver position with coarse-time assistance in $\mathrm{O} 2 \mathrm{O}$ scenario.

- In the coarse-time tests, the SNRs that are relative to the acquired satellites (depicted in Figure 10(b)) are lower than in the fine-time assistance case (depicted in Figure 10(a)),

- In the coarse-time tests the pseudorange rates are included in the geometry matrix, leading to an horizontal dilution of precision degradation [16].

For what concerns the computational load of the positioning procedure, it is important to note that the provision of the navigation message leads to a drastic reduction of the TTFF for the aided receiver in both coarse and fine time assistance.

7.3.2. Outdoor-to-outdoor $(\mathrm{O} 2 \mathrm{O})$ aiding scenario. The position results for both fine- and coarse-time assistance tests, are shown in Figure 12, while Table II reports the performance, which is relative to the East and North axes in terms of mean error and the standard deviation. Also in the $\mathrm{O} 2 \mathrm{O}$ scenario, the fine-time positioning is more accurate than the coarse-time one while the computational loads of the positioning procedure, either in presence of fine- or coarse-time assistances, are comparable with the ones of the $\mathrm{O} 2 \mathrm{I}$ case. As in the $\mathrm{O} 2 \mathrm{I}$ scenario, the provided information allows the aided receiver to take advantage of the TTFF reduction.

\section{CONCLUSION}

In this paper, we evaluated the benefits and the disadvantages of the cooperation between peers in the positioning algorithms, by focusing on the effects of the time synchronization on the acquisition and positioning procedures. In particular, we considered two GPS software receivers, which have 
been implemented in MATLAB and cooperate in both the O2I and the O2O scenarios. For each of them, we assessed the satellite signal acquisition effectiveness, the computational load in the signal acquisition procedure and the performance in terms of position accuracy and time-to-first fix in the positioning routine.

In the O2I aiding scenario, the indoor aided receiver has to improve its sensitivity, causing a computational load increase in the acquisition process, either in presence of fine or coarse time assistance. The positioning results confirm that the aided receiver position with fine-time is more accurate than the coarse-time.

In $\mathrm{O} 2 \mathrm{O}$ aiding scenario, the outdoor aided receiver does not need to strongly increase its sensitivity. Therefore, it can directly exploit the aiding information to reduce the TTFF, even in the acquisition process, either in presence of fine- or coarse-time assistance. The fine-time positioning is more accurate than coarse-time positioning (as in the O2I scenario) and the computational loads of the positioning procedures are comparable with the O2I scenario ones.

\section{ACKNOWLEDGMENTS}

This work has been supported by the Italian Research Programs (PON): 'TETRis: innovative Open Source Services over TETRA' and 'MC3-CARE: Mobile Continuous Connected Comprehensive - Care'.

\section{REFERENCES}

1. Barnes R, Winterbotton J, Dawson M. Internet geolocation and location-based services. IEEE Communications Magazine 2011; 49(4):102-108.

2. Kjærgaard MB. Location-based services on mobile phones: minimizing power consumption. IEEE Pervasive Computing 2012; 11(1):67-73.

3. Del Re E, Morosi S, Jayousi S, Sacchi C. SALICE Satellite-Assisted Locallzation and Communication systems for Emergency services. Proceedings of the 1st International Conference on Wireless Communications, Vehicular Technology, Information Theory and Aerospace and Electronic Systems (WVITAE), Aalborg, Denmark, 2009; 544-548.

4. Torres-Solis J, Falk TH, Chau T. A review of indoor localization technologies towards navigational assistance for topographical disorientation, Ambient Intelligence, intechopen.com, 2010.

5. Morosi S, Jayousi S, Falletti E, Araniti G. Cooperative Strategies in satellite assisted emergency services. International Journal of Satellite Communications and Networking 2013; 31(3):141-156.

6. Garello R, Presti LL, Corazza GE, Samson J. Peer-to-Peer Cooperative Positioning Part I: GNSS Aided Acquisition, Inside GNSS, 2012.

7. Dovis F, Chiasserini CF, Musumeci L, Borgiattino C. Context-aware peer-to-peer and cooperative positioning. International Conference on Localization and GNSS (ICL-GNSS), Helsinki, Finland, 2014; 1-6.

8. Xiong Z, Sottile F, Spirito MA, Garello R. Analysis of hybrid and cooperative positioning algorithms in urban canyon scenarios. International Conference on Localization and GNSS (ICL-GNSS), Turin, Italy, 2013; 1-6.

9. Liu Y, Tian S, Li G, Dai W, Chang J. On the localizability of cooperative positioning: two user model. International Conference on Wireless Communications and Signal Processing (WCSP), Hefei, China, 2014; 1-5.

10. Borre K, Akos DM, Bertelsen N, Rinder P, Jensen SH. A software-Defined, GPS and Galileo Receiver: A single-Frequency Approach. Birkhuser: New York, 2007.

11. Defraigne P, Banerjee P, Lewandowski W. Time transfer through GPS. Indian Journal of Radio and Space Physics 2007; 36:303-312.

12. Neaogoe T, Cristea V, Banica L. NTP versus PTP in computer network clock synchronization. IEEE ISIE 2006.

13. Morosi S, Del Re E, Martinelli A. P2P Cooperative GPS Positioning with Fine/Coarse Time Assistance. Proceedings of the ICL-GNSS, Torino, Italy, 2013; 1-6.

14. Morosi S, Del Re E, Martinelli A. Cooperative GPS Positioning with Peer-to-Peer Time Assistance. Proceedings of the 4th International Conference on Wireless Communications, Vehicular Technology, Information Theory and Aerospace and Electronic Systems (WVITAE), Aalborg, Denmark, 2014; 1-5.

15. Kalamandeen A, Scannell A, de Lara E, Sheth A, LaMarca A. Ensemble: Cooperative Proximity-based Authentication. MobiSys10, San Francisco, California, USA, 2010; 331-344.

16. Diggelen FV. A-GPS: Assisted GPS, GNSS, and SBAS (1st ed.). Artech House: Boston, MT, USA, 2009.

17. Lo Presti L, Margaria D, Samson J. A novel Peer to Peer Acquisition Strategy Tailored to Galileo E1 R. 53nd International Symposium ELMAR, Zadar, Croatia, 2010; 417-424.

18. Caceres MA, Penna F, Wymeersch H, Garello R. Hybrid Cooperative Positioning Based on Distributed Belief Propagation. IEEE Journal on Selected Areas in Communications 2011; 10:29.

19. Robinson M. Topological localization via signals of opportunity. IEEE Transactions on Signal Processing 2012; 60:5.

20. Petovello M, Muthuraman K. GNSS Solutions: Coarse Time Positioning. Inside GNSS: Eugene, OR, USA, 2012.

21. Diggelen FV. Method and Apparatus for Time Free Processing of GPS Signals 2002. 


\section{COOPERATIVE GPS POSITIONING}

22. Audoin C, Guinot B. The Measurements of Time - Time, Frequency and the Atomic Clock. Cambridge University Press: Cambridge, UK, 2001.

23. Jin S. Global Navigation Satellite Systems, Signal, Theory and Application. Intech: Rijeka, Croatia, 2012.

24. Holmes JK. Spread Spectrum Systems for GNSS and Wireless Communications. Artech House: Boston, MT, USA, 2007.

25. Krasner N. Method and Apparatus for Determining Time for GPS Receivers,? U.S. Patent 6,150, 980, 1999.

26. (Available from: http://www.spurkfun.com/products/10981) [Accessed date on 21 December 2014].

27. Groves PD. A-GPS: Principle of GNSS, Inertial and Multisensor Integrated Navigation Systems (2st ed.). Artech House: Boston, MT, USA, 2013.

\section{AUTHORS' BIOGRAPHIES}

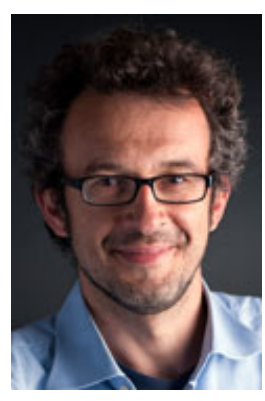

Simone Morosi received his Ph.D. degree in information and telecommunication engineering from the University of Florence in 2000. He is currently an assistant professor at the same university. His present research interests focus on communication systems, green ICT and energy-efficient wireless communications, positioning techniques, and future wireless communication systems. He is an author of more than 100 papers in international journals and conference proceedings.

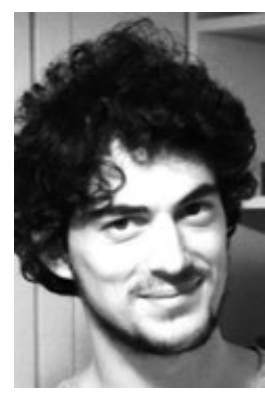

Alessio Martinelli received his Master's degree in Telecommunication Engineering from the University of Florence in 2013. He is currently a Ph.D. student in Information Engineering at the Department of Information Engineering of the University of Florence. His research activity is mainly focused on positioning and navigation solutions: cooperative GNSS Positioning, GNSS/INS integration, pedestrian movement recognition and classification, pedestrian dead reckoning (PDR) navigation.

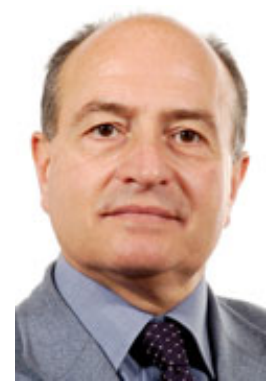

Enrico Del Re is a full professor at the University of Florence, Florence, Italy. Presently, he is the Director of the Department of Information Engineering (DINFO) of the University of Florence and President of the Italian Interuniversity Consortium for Telecommunications (CNIT), having served before as Director. He is the head of the Signal Processing and Communications Laboratory of the Department of Information Engineering (DINFO) of the University of Florence. 